\title{
Online Diagnostic Lab Reporting System
}

\section{S. N. Shitole, Girish Kumar, Kiran Devesh K. Singh, Leena Boudhkumar Khobragade}

Department of Computer Engineering, MIT Polytechnic, Pune, Maharashtra, India

\begin{abstract}
Article Info

Volume 7, Issue 3

Page Number: 638-640

Publication Issue :

May-June-2021

\section{Article History}

Accepted : 10 June 2021

Published : 18 June 2021

The determination of the project capacitates as "ONLINE DIAGNOSTIC LAB REPORTING SYSTEM" is to develop a web application to computerize the Management of a Diagnostic system that is user -friendly simple, and costeffective. It comes with the collection of information of patients, details of the diagnosis, etc. The main function of this system is firstly registered and then store the details of the patient and the details of the staff and get back these details as and when required, and also for the utilize these details Purposely. System input holds the Patient's details, diagnosis details, while system output is to get these details on the screen. The Online Diagnosis Lab Reporting system can be pass into using a username and password. And it is accessible by each level of a user according to their part. Every user can see this data that they are approachable. And only that user can add the data into the database who has editing permission. The data can be recovers easily. The data are efficiently protected for personal use and makes clarifying of data very rapidly.
\end{abstract}

Keywords: Online Diagnostic, Diagnostic Centers

\section{INTRODUCTION}

Presently, in our country, most of our Diagnostic Centers use a manual system for the management and preservation of censorious information. The current system requires many paper forms with data stores spread throughout the diagnostic center management framework Often Information on the forms is insufficient or to stick to management standards. Forms are often lost in conveyance between departments requiring a Complete auditing process to ensure that no necessary information is lost. Multiple Copies of the Same information exists in the diagnostic center and may lead to unpredictability data in various data stores. the hospital daily work panniers in that Conventional way.

The purpose of this project is to design an online diagnostic lab manager application that brings up various diagnoses that are working online. and its main aim is to bring together various diagnostic working, researches on one single platform that is also online (so that it can be accessible for everyone).

This website turns manual tasks automatically to save recourses. Automatic works are considered more trustful, reliable, accurate, perfect, etc. The proposed system is an online system which is preferably a website and management system together. The

Copyright: @ the author(s), publisher and licensee Technoscience Academy. This is an open-access article distributed under the terms of the Creative Commons Attribution Non-Commercial License, which permits unrestricted non-commercial use, distribution, and reproduction in any medium, provided the original work is properly cited 
purpose of this project is to provide faster and reliable and comfortable service as compared to manual systems. This system may increase the profit for diagnostic labs. The user-friendly and interactive interface makes using this application easy for and everyone. Users/patients register themselves after that online providing their basic details and select the tests that they want to perform under that particular lab. The system is usable, reliable, and maintainable too.

\section{LITERATURE SURVEY}

Health is imperative to everyone. Health can't be bought by anything. Health is precious no matter what country we are from or what race we are, or what age or gender we have some important details like the patient's allergy and medical history will be neglected by the doctors when they diagnosing. The strategy for diagnosing also Devours amount of time although the patients come with a retail problem that they can settle by themselves if they have the right help. This paper discusses the Web-Based Online Medical Diagnosis system.

In a pathology laboratory, supplementary appointments are a time-consuming and manual process, which is facing Downward to human errors There is also the fact that this traditional way of managing pathology laboratories is very unsuitable for the patients since they have to go to the lab to get an appointment and then to wait for their number and then give a sample for testing.

And when the reports are ready, they again have to go to the lab to retrieve them. Our system makes it very easy and convenient for the patients since it removes the need to especially for old people. go to the lab in most cases.
The engagements are scheduled online, the sample is collected from the patient's place and the reports are accessible to the patient online.

As you initiate our online Diagnostic Lab Reporting system, we can say that this structured system will ensure excellence daily work routine of any diagnostic center and also reinstate the compatible performance of the staff due to the well-ordered, marshal approach of our system.

\section{PROPOSED METHODOLOGY}

Diagnostic System is designed for any diagnostic center to supplant their subsisting manual paperbased system. The new system is to control the information of patients, testing capability, and patient debited. These services are to be provided in an efficient, cost-effective manner, to reduce the time and the resources currently needed for such tasks. This system provides online storage or the updating and salvage facility. This system promises very less or no paperwork and also provides help to the operational staff.

In this system, everything is stored electronically so a very less amount of paperwork is required and information can be retrieved very easily without searching here and there into registers. This system is been deliberate here.

\section{IMPLEMENTATION}

Design is the first step in the accretion phase for any techniques and principles to define a device, a process, or system in sufficient detail to Tolerate its physical completeness. Once the software requirements have been analyzed and specified, the software design involves three technical activities - design, coding, and implementation, and testing that is required to build and corroborate the software. The design 
activities are of main importance in this phase because, in this activity, decisions in conclusion affecting the success of the software implementation and its case of maintenance are created. These decisions have the final conducting on the reliability and maintainability of the system.

Design is the only way to rigorously translate the customer's need into finished software or a system. Design is the place where quality is encouraged in development. Software design is a process through which requirements are translated into a suggestion of the software. Software design is directed in two steps. Introductory design is United with the transformation of requirements into data.
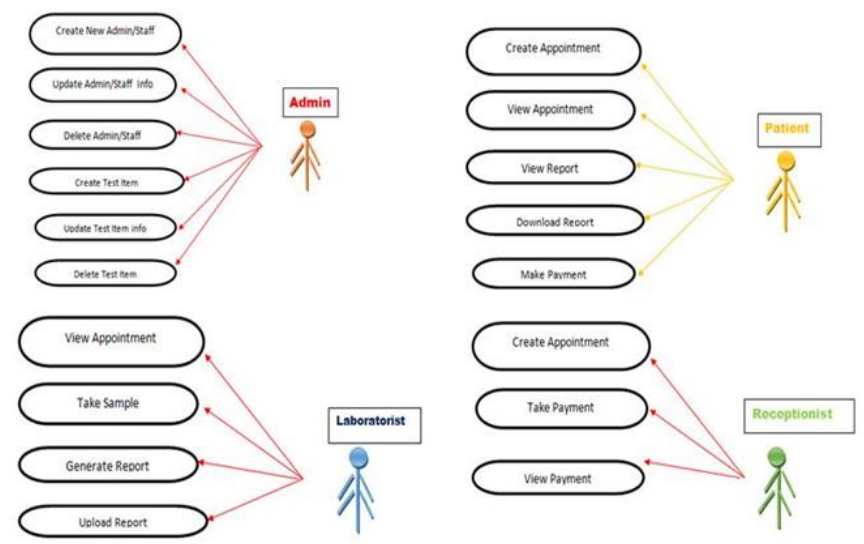

Fig 1. Use Case

\section{CONCLUSION}

Since we are entering details of the patients and their diagnostic information electronically in our "Online Diagnostic Lab Reporting System", data will be fixed. Using this application, we can recover a patient's history with a single click. Thus, processing information will be faster. It guarantees that the accurate maintenance of the patient details. It easily reduces the bookkeeping task and thus Reduces the human effort and increases accuracy speed. The system also provides the facility of trig as per the requirement. It will be able to scope for Everson in the future if it is necessary.

It will be able to Reduce potential errors in regulatory Assent, operational processes, and patient safety in the diagnostic system.

\section{REFERENCES}

[1]. https:/github.com/shihabhub/OnlineDiagnostic-Lab-Reporting-System

[2]. https://nevonprojects.com/online-diagnosticlab-reporting-system-

php/\#: :text=It\%20is\%20an\%20online\%20diag nostic,and\%20login\%20using\%20registered\%2 Odetails.\&text=The $\% 20$ system $\% 20$ allows $\% 20 \mathrm{ad}$ min\%20to,email\%20it\%20to\%20intended $\% 20$ patient.

[3]. https://www.academia.edu/43044163/ONLINE _DIAGNOSTIC_LAB_REPORTING_SYSTEM

[4]. https://pdfcoffee.com/online-diagnostic-labreporting-system-pdf-free.html

[5]. https://m.facebook.com/finalyearproject.info/p hotos/a.156786468016585/344217985940098/?t ype $=3$

\section{Cite this article as :}

S. N. Shitole, Girish Kumar, Kiran Devesh K. Singh, Leena Boudhkumar Khobragade, "Online Diagnostic Lab Reporting System", International Journal of Scientific Research in Computer Science, Engineering and Information Technology (IJSRCSEIT), ISSN : 2456-3307, Volume 7 Issue 3, pp. 638-640, May-June 2021. Available at

doi : https://doi.org/10.32628/CSEIT217150 Journal URL : https://ijsrcseit.com/CSEIT217150 